\title{
Characterization of Inkjet-printed Digital Microfluidics Devices
}

\author{
Shiyu Chen \\ Graduate Student \\ Department of Mechanical Engineering, \\ University of Washington, Seattle, USA \\ Zhidong $\mathrm{He}$ \\ Graduate Student \\ Department of Mechanical Engineering, \\ University of Washington, Seattle, USA \\ Suhwan Choi \\ Post-doctoral Research Associate \\ Department of Mechanical Engineering, \\ University of Washington, Seattle, USA \\ Igor V Novosselov \\ (Corresponding Author) \\ Research Associate Professor, \\ Department of Mechanical Engineering, \\ University of Washington, Seattle, USA \\ Email: ivn@uw.edu
}

\begin{abstract}
Digital microfluidics (DMF) devices enable precise manipulation of small liquid volumes in point-of-care testing. Printed circuit board (PCB) substrate is commonly utilized to build DMF devices. However, inkjet printing can be used to fabricate DMF circuits, providing a less expensive alternative to PCB-based DMF designs while enabling more rapid design iteration cycles. We demonstrate the fabrication process of the inkjet-printed DMF circuit. We compare the use of Kapton and polymethyl methacrylate (PMMA) as a dielectric by measuring the minimal droplet actuation voltage for a range of actuation frequencies. The minimum actuation voltage of $5.6 \mathrm{~V}$ was required for droplet movement with the PMMA layer thickness of $0.2 \mu \mathrm{m}$ and a hydrophobic layer of $0.17 \mu \mathrm{m}$. Significant issues with PMMA dielectric breakdown were observed at actuation voltages above $10 \mathrm{~V}$. In comparison, devices that utilized Kapton were found to be more robust even at the actuation voltage up to $100 \mathrm{~V}$.
\end{abstract}

\section{Introduction}

Digital microfluidics (DMF) is an approach for manipulating small liquid volumes using electrostatic force; it allows for precisely controlling the individual droplet movement in microliter to nanoliter volume on an array of electrodes [1]. DMF can perform droplet operations such as dispensing, mixing, splitting, and merging without external sources like pumps and valves that channel-based microfluidics require [2]. Complex multistep operations (e.g., washing, solvent exchanges, and extractions) can also be automated utilizing generic device geometry. Typically, there are two common configurations of DMF devices: oneplate and two-plate devices. In the one-plate format, droplets are manipulated on a single substrate bearing both actuation and ground electrodes. In the two-plate format, droplets are sandwiched between a counterelectrode affixed on the top plate and a bottom plate bearing an array of insulated driving electrodes. Twoplate devices can implement more operations, most notably dispensing and splitting [3]. Two-plate DMF 
devices can be operated in air or silicone oil $[4,5]$. Although the operation procedures in an open system are more straightforward, lower actuation voltages can be used due to a reduction in surface tension in an oil-immersed system; evaporation of droplets is also eliminated [3].

Fabrication of DMF devices is challenging and is often complicated by the need for a cleanroom. A common way to manufacture DMF chips is to utilize the printed circuit boards (PCB) because of their relatively low production cost and multilayer format, enabling complex electrode and wiring design [3, 4]. However, the deposition of dielectric and hydrophobic layers onto PCB-DMF is tedious and time-consuming; thus, the preproduction design cycle of PCB-DMF can be rather long. Inkjet printing and other deposition methods have been utilized for microfluidic devices due to their low cost. They are suitable for mass production, shortening the fabrication time and the design cycle $[5,6]$. Novel printing and coating methods have recently been reported for wearable devices, e.g., refs [7-10]

In DMF devices, electrodes first are covered with a dielectric layer; then, a hydrophobic coating is applied to ensure smooth droplet movement [11]. The effects of coating thickness, deposition uniformity, high initial contact angle, low contact angle hysteresis should be considered when choosing the hydrophobic material [12]. The common materials include Teflon, Parafilms, FluoroPel, Cytop, and polydimethylsiloxane (PDMS) [13]. These materials' thicknesses vary from $50 \mu \mathrm{m}$ to $100 \mu \mathrm{m}$, and the actuation voltages may vary from $50 \mathrm{~V}$ to $800 \mathrm{~V}$ [14-16]. The actuation voltages are primarily affected by the dielectric layer's thickness, and materials may fail when the applied voltage exceeds a limit [17]. Polymeric and inorganic materials are widely used in manufacturing dielectric layers. Parylene-C is often used as an insulating coating in DMF because of its relatively high dielectric constant and lower voltage that changes the contact angle [17]. Silicon dioxide ( $\mathrm{SiO} 2)$ can serve as an inorganic insulator due to its high dielectric constant and strength [18]. Recently, PMMA and Kapton tape have been used as dielectric layers in DMF. The main advantages are their low cost, simple manufacturing process, and low thickness that can translate to lower droplet actuation voltage. Parametric characterization of these coatings is required to advance the fabrication of the low-cost DMF devices. The stability of these materials under repeating exposure to high electric fields also needs investigation.

This paper presents a methodology for fabrication of DMF circuit using inkjet printer followed by coating by dielectric and hydrophobic materials. A simple 5-electrode DMF cartridge is used to demonstrate droplet manipulation. Two dielectrics, Kapton and PMMA, were tested in terms of droplet actuation frequency and minimum actuation voltage. All fabrication steps were performed outside the cleanroom enabling rapid, low-cost DMF chip fabrication.

\section{Experimental methods}

The materials, equipment, and detailed fabrication steps used in the IJP DMF chips fabrication are specified. Coatings characterization and droplet manipulation procedures are described in this section.

\subsection{Material and Equipment}

The inkjet-printed DMF circuit was fabricated using Fujifilm Dimatix Material Printer (DMP) by depositing silver nanoparticles ink (Ag ink) onto polyethylene terephthalate (PET) film. The thickness of the PMMA dielectric layer and the hydrophobic layer on Kapton ${ }^{\circledR}$ tape was measured with an optical profilometer (Olympus OLS41) with 100x magnification.

The power source consisted of a function generator (BK Precision 4052, Yorda Linda, CA ) and a high voltage (HV) amplifier (Trek PZ700, Advanced Energy, Dever, CO). A digital I/O board DAQpad-6229 BNC, National Instruments Co. (NI, Fort Collins, CO) was used to energize the relays (AQW614EH, Panasonic, Osaka, Japan) linked to the individual electrode. The system was controlled by custom LABVIEW code (NI, Fort Collins, CO) 
Top covers are glass slides with ITO coated PET film with surface resistivity $60 \Omega /$ sq, (Sigma Aldridge, St Louis, MO) that surfed as a counter electrode. These substrates were cleaned by plasma cleaner before spin coating. Spin coater was used to deposit the dielectric and hydrophobic layers. Dielectric materials included Kapton or $0.4 \%, 1.5 \%$, and $4 \%$ by weight of PMMA in anisole (Sigma Aldridge, St Louis, MO). FluoroPel PFC1101V (Cytonix, Beltsville, MD) was used to apply the hydrophobic layer. Silicone oil of 1 CST (Sigma Aldridge, St Louis, MO) was applied as filler media in the cartridge.

\subsection{Device Fabrication}

The DMF chips fabrication protocol consisted of printing DMF electrodes applying coatings to the top and bottom plates, and assembling the cartridge. Figure 1 shows the conceptual diagram of the assembled device.

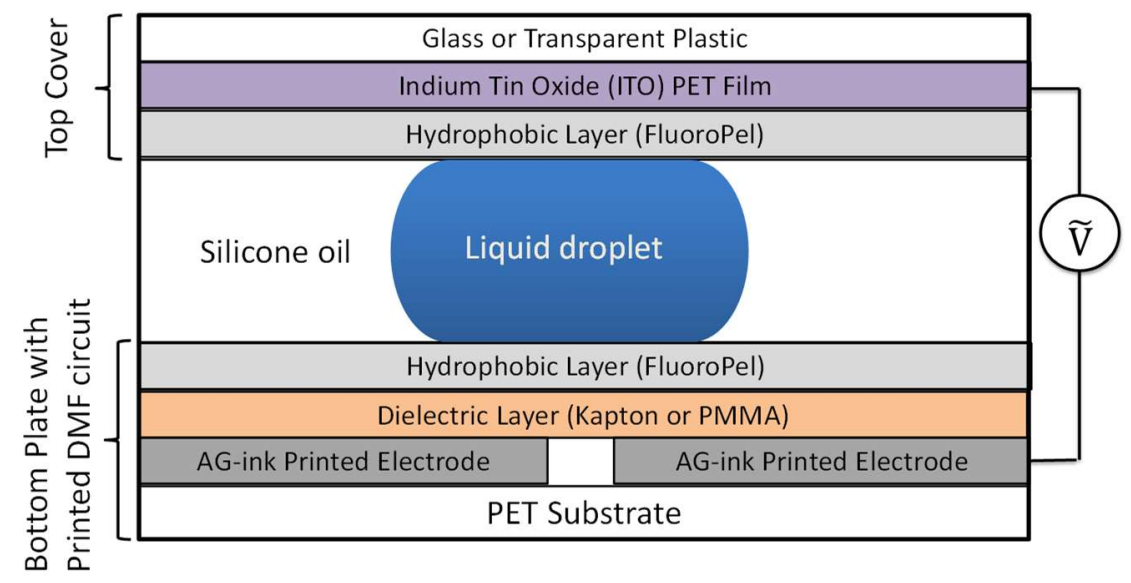

Figure 1: Conceptual diagram showing the assembly layers of the DMF cartridge with Ag ink printed electrodes. The schematic is not drawn to scale.

\subsubsection{Printing Electrodes}

The DMF circuits were printed using a DMP-2800 with silver nanoparticle ink (Ag ink) (Sigma Aldridge, St Louis, MO). The DMP settings were 22-volt firing voltage, 700-micrometer print height, 25-micrometers drop spacing, single printing nozzle was used. As suggested by the previous report, to achieve low electrical resistivity of Ag ink [19], the printed samples were UV cured for 480 seconds. Figure 2 shows the printed circuit on PET substrate; the design consists of one larger electrode for droplet introduction and four smaller electrodes $1.5 \mathrm{~mm} \times 1.5 \mathrm{~mm}$ for droplet transport. The electrodes are separated by a $100 \mu \mathrm{m}$ gap. Each electrode is connected to a $1.8 \mathrm{~mm} \times 1.8 \mathrm{~mm}$ pad for interface with power supply via a $100 \mu \mathrm{m}$ - width lead. 


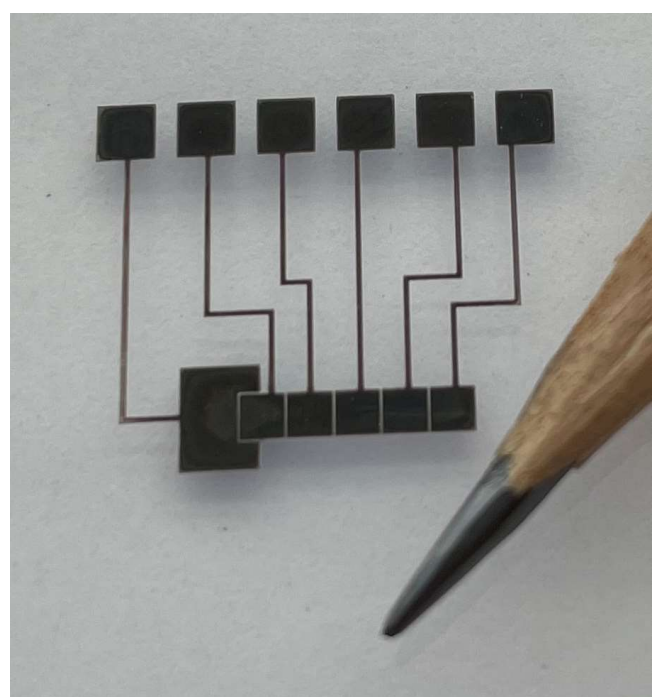

Figure 2. Printed DMF circuit on PET substrate.

\subsubsection{Cartridge Fabrication}

The bottom plate consists of a glass coverslip, a PET substrate with the printed circuit, a dielectric layer, and a hydrophobic layer. The top cover consists of a glass slide, ITO PET film, and a hydrophobic layer. The diameter of the holes is compatible with a standard pipette tip.

Surface defects such as dust or scratches can seriously affect the droplet movement; therefore, the Plasma cleaner was used to clean the PET substrate with the printed circuit. The PET substrate was attached to the glass coverslip with the double-sided adhesive tape.

Before applying the PMMA layer, the contact pads were masked by adhesive tape to prevent PMMA deposition on the electrical contacts. For bottom plate fabrication, $100 \mu \mathrm{L}$ of PMMA reagent was used. The spin coater speed was set to $500 \mathrm{rpm}$ for 10 seconds, followed by $1500 \mathrm{rpm}$ for 30 seconds. After drying at room temperature for 20 minutes, $100 \mu \mathrm{L}$ of Fluoropel solution was applied, spin coater was set at $500 \mathrm{rpm}$ for 10 seconds followed by $1750 \mathrm{rpm}$ for 30 seconds. The top cover ports were drilled from the PET side to avoid breaking and chipping the glass. Before assembly, all parts were dusted with clean compressed air.

The precut silicon gasket was fixed to the bottom plate with UV-sensitive adhesive. The adhesive is cured in a UV box for 10 minutes. The corners between the gasket and bottom plate were touched with the hydrophobic solution to fill any gaps. The top cover was glued to the bottom plate, and the entire assembly is UV cured for 30 minutes. Before the droplet test, the cartridge was filled with silicone oil to reduce the surface tension between the droplet and the components; then a $1 \mu \mathrm{L}$ droplet was injected through the injection port, see Figure 3. 


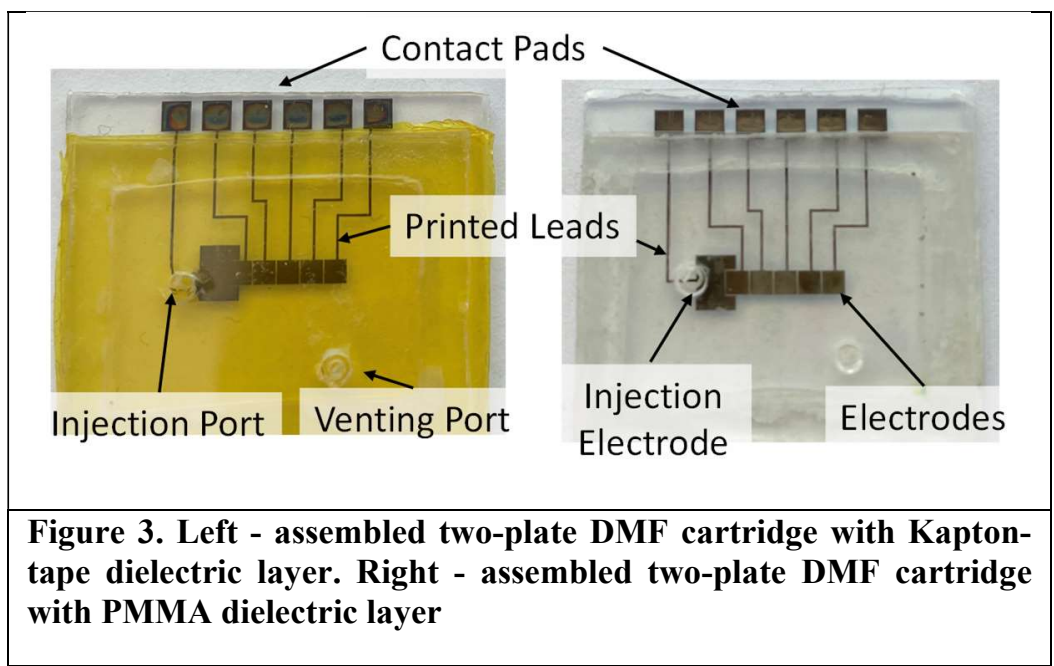

\subsection{Device Operation}

To operate the DMF circuit, the actuation signal was applied to the electrodes. The voltage was varied to determine the minimum operating potential for each droplet movement frequency. Figure 4 shows the diagram of the experimental setup. The control circuit was driven by a LABVIEW program to pass the actuation voltage to the specific electrodes. We evaluated droplet movement frequency $(f)$, defined by the number of times a droplet moves back and forth between two electrodes per second. The frequency was set to $f=0.2 \mathrm{~Hz}, 1 \mathrm{~Hz}, 2 \mathrm{~Hz}, 5 \mathrm{~Hz}$ by the computer. For each frequency, a range of voltages was applied $\varphi=10$ $-300 \mathrm{~V}$.

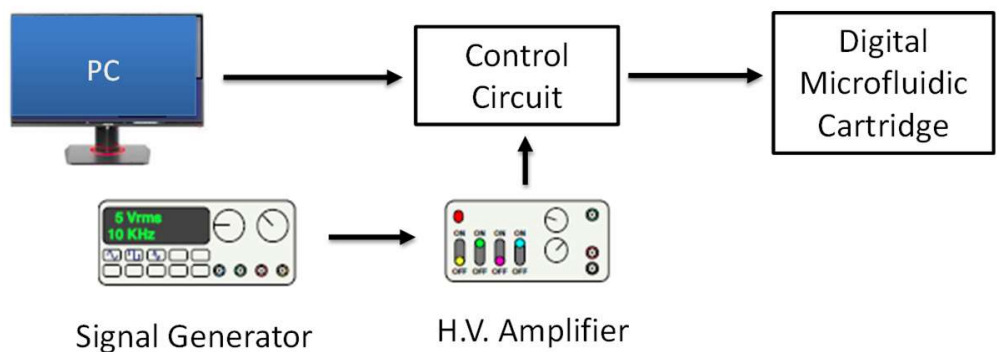

Figure 4. Diagram of DMF system. The function generator signal is amplified in the high voltage amplifier and passed through the control circuit to a specific electrode.

\section{Results and Discussion}

\subsection{Coating Thickness Evaluation}

Coating thickness was evaluated as a function of deposition parameters. Figure 5 (a) shows the thickness of the PMMA layer against the PMMA concentration in the anisole solution. The PET substrate thickness was measured before the coating procedure and again after coating. To evaluate the repeatability of the procedure, three batches for each concentration were prepared. The thickness of PMMA samples ranges from $0.17 \pm 0.05 \mu \mathrm{m}$ to $1.97 \pm 0.14 \mu \mathrm{m}$. The thickness increases with the weight percentage of the PMMA in the solution. The required actuation voltage is lower for thinner dielectric layers; however, the thinner dielectric layer leads to the dielectric breakdown, limiting the actuation voltage range. 
The effect of rotational speed on FluoroPel deposition thickness was studied; the thinner layer is desired to reduce the cost of reagents. Figure 5 (b) shows the thickness of hydrophobic layers coated on Kapton tape with five different revolution speeds. The thickness of Kapton tape was measured before and after the coating to calculate the deposition thickness. The Fluoropel layer thickness decreases as the rotational speeds increase. The hydrophobic layer's target thickness was $\sim 0.2 \mu \mathrm{m}$, so the speeds $>1500 \mathrm{rpm}$ are acceptable. Considering the similar thicknesses for $1750 \mathrm{rpm}$ and $2000 \mathrm{rpm}, 1750 \mathrm{rpm}$ was chosen for the cartridge fabrication.

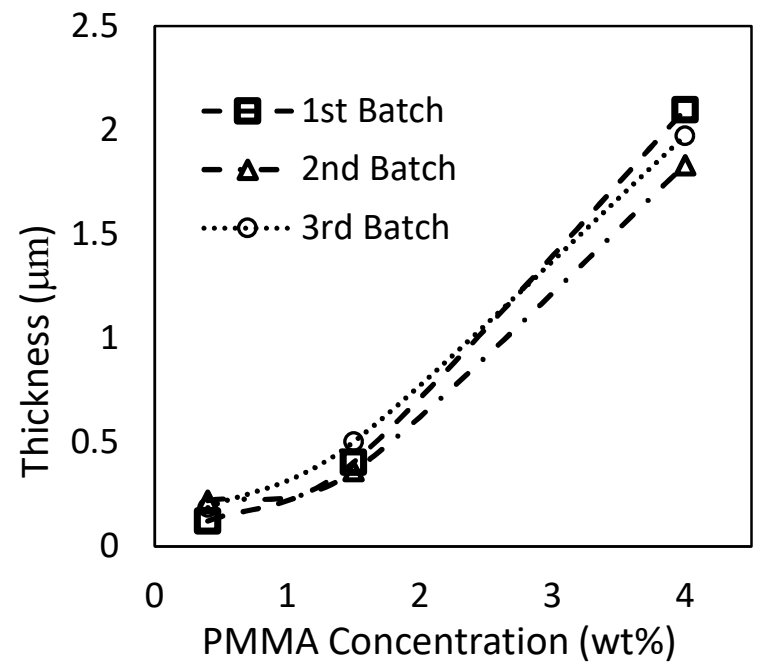

(a)

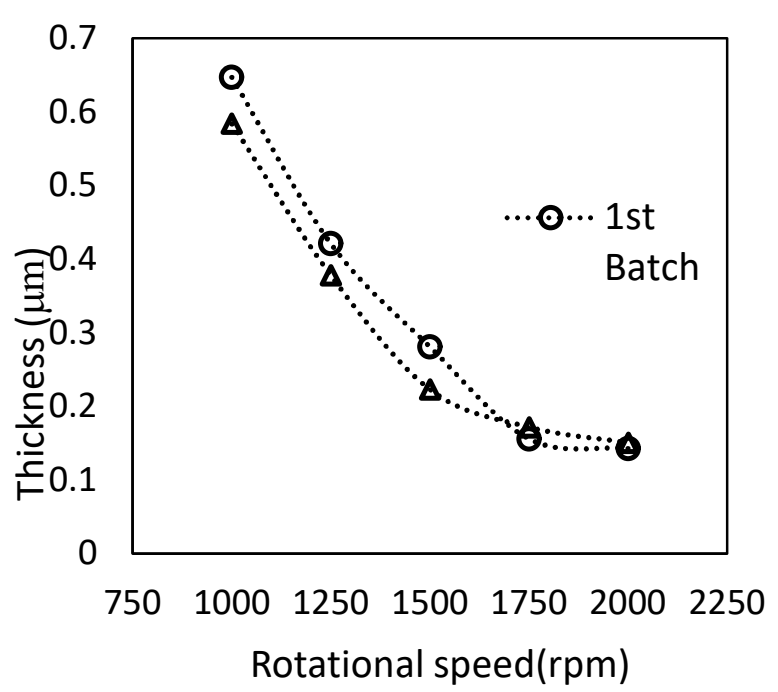

(b)

Figure 5. (a) Thickness of the PMMA layer as a function of PMMA concentration in anisole $(0.4 \%, 1.5 \%$, and $4 \%$ ) the coater rotational speed was set to 1750rpm. (b) Thickness of Fluoropel layer for spin coater setting of 1000rpm, 1250, 1500rpm, 1750rpm, and 2000rpm on Kapton.

\subsection{Droplet movement evaluation}

Both PMMA and Kapton cartridges were tested. In each experiment, water droplet $(1 \mu \mathrm{L})$ colored by the food coloring was injected into the cartridge, and the voltage was applied to the DFM circuit. The video demonstrating droplet movement is presented as Supplemental Information.

In the Kapton DMF cartridge experiments, 3 different chips were tested at 4 different frequencies. Figure 6 (a) shows that all three samples have similar characteristics, i.e., minimal actuation voltages vs. actuation frequency are nearly identical for all cartridges. As the input frequency increases, the system required higher voltages to oscillate the droplet. For $f=0.2 \mathrm{~Hz}$, the minimum voltage was $27 \mathrm{~V}$; for $f=5 \mathrm{~Hz}$, the minimal actuation voltage was $85 \mathrm{~V}$. The increase of the required voltage at high frequencies points to the system's hysteresis, i.e., the system needs to re-equilibrate after the initial droplet movement and before the movement opposite direction can occur. At the higher frequency, higher Coulombic forces are required to overcome the low angle hysteresis, inertia, surface tension, and other physiochemical interaction associated with the liquid-surface-charge interaction. Detailed analysis of this transient regime requires further investigation, and it is beyond the scope of this manuscript.

PMMA coated DMF cartridges with 3 different PMMA thicknesses were tested $(0.17 \mu \mathrm{m}, 0.43 \mu \mathrm{m}, 1.97$ $\mu \mathrm{m})$. Figure 6 (b) shows the minimum voltage required to manipulate the droplet as a function of actuation frequency. As expected, the devices prepared with lower PMMA concentrations (thinner dielectric layers) required lower actuation voltages. For $0.2 \mathrm{~Hz}$, the PMMA coated samples only needed around 6 volts to initiate the droplet transport. The maximum required voltage was $\sim 20 \mathrm{~V}$ at $5 \mathrm{~Hz}$ and $2 \mu \mathrm{m}$ PMMA thickness (4\% concentration). However, the dielectric breakdown was observed for PMMA coated chip. At $\varphi>10$ 
$\mathrm{V}$, the bubbles formed around the energized electrode. The bubbles completely filled up space around the droplet blocking it from moving at $\varphi>20 \mathrm{~V}$.

The PMMA layer was significantly thinner $(0.2 \mu \mathrm{m}-2 \mu \mathrm{m})$ compared to the Kapton layer $(\sim 30 \mu \mathrm{m})$. The electrical field strength for Kapton DMF at the droplet interface is lower for the same actuation voltage. Thus, to reach threshold for droplet movement, a higher actuation voltage is required.

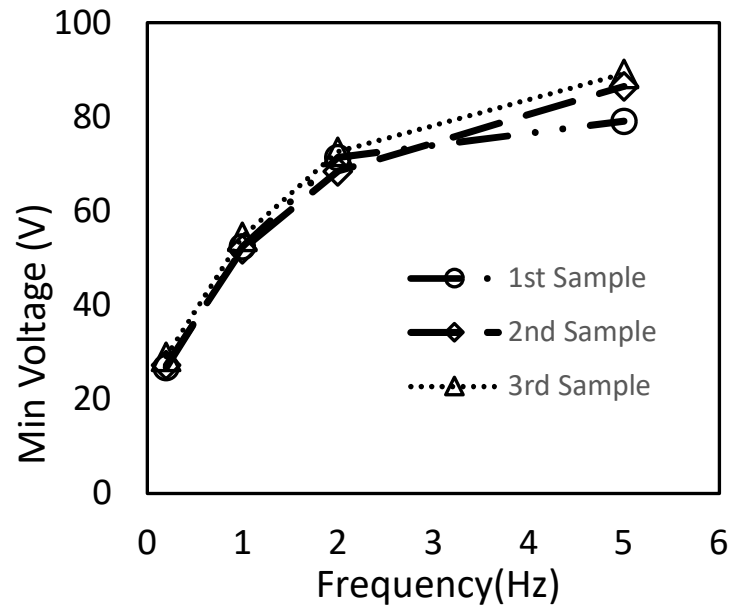

(a)

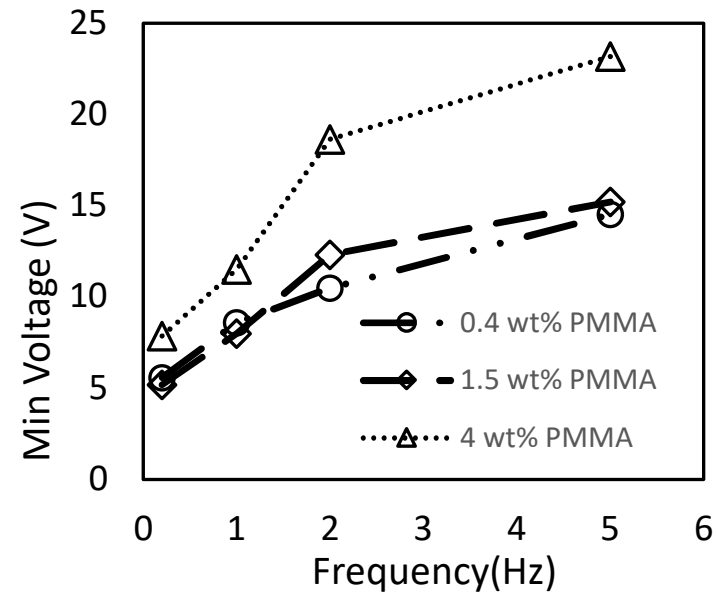

(b)

Figure 6. Minimal droplet actuation voltages for $f=0.2 \mathrm{~Hz}, 1 \mathrm{~Hz}, 2 \mathrm{~Hz}$, and $5 \mathrm{~Hz}$. (a) Three identical Kapton DMF devices are tested, (b) PMMA DMF for three different recipes, i.e., different dielectric thicknesses

\section{Conclusions}

This communication presents proof-of-concept experiments for inkjet-printed DMF circuits. We report the fabrication method for a simple DMF chip. In the chips' fabrication, we employed two different dielectric layers: Kapton $(\sim 30 \mu \mathrm{m})$ and PMMA $(0.2 \mu \mathrm{m}-2 \mu \mathrm{m})$. FluoroPel coating was used as a hydrophobic layer. Low dielectric thicknesses of PMMA coating enabled low thresholds for droplet manipulation. In the case of $0.2 \mu \mathrm{m}$ PMMA layer followed by $0.17 \mu \mathrm{m}$ FlouroPel the actuation threshold was $\sim 5.6 \mathrm{~V}$. For the Kapton DMF device, the minimum actuation voltage was $\varphi=26.8 \mathrm{~V}$. In terms of durability, PMMA coated circuit showed bubble formation at the energized electrode which blocked droplet movement. The Kapton-coated printed circuit was more robust. Specific attention should be paid to the dielectric layer's effect on perfomance of DMF devices in future designs.

Funding: This research was funded by the National Institute of Health grants U01 EB021923 and NIEHS R42ES026532 subcontract to the University of Washington. Printing of the DMF circuits was conducted at the Washington Clean Energy Testbeds, an open-access research facility operated by the University of Washington Clean Energy Institute. Surface characterization was performed in a Washington Nanotechnology Center, a National Nanotechnology Coordinated Infrastructure site at the University of Washington, supported in part by the National Science Foundation, grant NNCI-1542101.

\section{References}

1. Abdelgawad, M. and A.R. Wheeler, The digital revolution: a new paradigm for microfluidics. Advanced Materials, 2009. 21(8): p. 920-925.

2. Cho, S.K., H. Moon, and C.-J. Kim, Creating, transporting, cutting, and merging liquid droplets by electrowetting-based actuation for digital microfluidic circuits. Journal of Microelectromechanical systems, 2003. 12(1): p. 70-80. 
3. Choi, K., et al., Digital microfluidics. Annual review of analytical chemistry, 2012. 5: p. 413-440.

4. Sista, R., et al., Development of a digital microfluidic platform for point of care testing. Lab on a Chip, 2008. 8(12): p. 2091-2104.

5. Dixon, C., et al., An inkjet printed, roll-coated digital microfluidic device for inexpensive, miniaturized diagnostic assays. Lab on a Chip, 2016. 16(23): p. 4560-4568.

6. Andò, B., et al., Low-cost inkjet printing technology for the rapid prototyping of transducers. Sensors, 2017. 17(4): p. 748.

7. Wang, H. and L. Chen, Electrowetting-on-Dielectric Based Economical Digital Microfluidic Chip on Flexible Substrate by Inkjet Printing. Micromachines, 2020. 11(12): p. 1113.

8. Joshi, K., V. Velasco, and R. Esfandyarpour, A Low-Cost, Disposable and Portable InkjetPrinted Biochip for the Developing World. Sensors, 2020. 20(12): p. 3593.

9. Fan, Y., et al., Low-cost and flexible film-based digital microfluidic devices. Micro \& Nano Letters, 2020. 15(3): p. 165-167.

10. Kahng, S.-J., et al., Nanoink bridge-induced capillary pen printing for chemical sensors. Nanotechnology, 2018. 29(33): p. 335304.

11. Gao, J., et al., Adhesion promoter for a multi-dielectric-layer on a digital microfluidic chip. RSC Advances, 2015. 5(60): p. 48626-48630.

12. Tabatabaeipour, G., H. Hajghassem, and M. MohtashamiFar, Low-cost hydrophobic layer as a top plate in two-plate digital microfluidics. European Physical Journal. Applied Physics, 2015. 71(1).

13. Koo, B. and C.-J. Kim, Evaluation of repeated electrowetting on three different fluoropolymer top coatings. Journal of Micromechanics and Microengineering, 2013. 23(6): p. 067002.

14. Ahmadi, A., et al., Electrohydrodynamic modeling of microdroplet transient dynamics in electrocapillary-based digital microfluidic devices. Microfluidics and nanofluidics, 2011. 10(5): p. 1019-1032.

15. Caputo, D., et al., Polydimethylsiloxane material as hydrophobic and insulating layer in electrowetting-on-dielectric systems. Microelectronics Journal, 2014. 45(12): p. 16841690.

16. Feng, J.-T. and Y.-P. Zhao, Experimental observation of electrical instability of droplets on dielectric layer. Journal of Physics D: Applied Physics, 2008. 41(5): p. 052004.

17. Liu, H., et al., Dielectric materials for electrowetting-on-dielectric actuation. Microsystem technologies, 2010. 16(3): p. 449.

18. Moon, H., et al., Low voltage electrowetting-on-dielectric. Journal of applied physics, 2002. 92(7): p. 4080-4087.

19. Ning, H., et al., UV-cured inkjet-printed silver gate electrode with low electrical resistivity. Nanoscale research letters, 2017. 12(1): p. 1-7. 